\title{
Drilling Holes Through Balls and Cubes*
}

\author{
Maximizing the Total Exposed Surface Area
}

\author{
Jaskirat Kaur, Jasmeen Lally and Jyotirmoy Sarkar
}

\begin{abstract}
Suppose that we are allowed to drill a cylindrical hole of any radius through the center of a unit ball or a unit cube in a given direction. Which radius will maximize the total exposed surface area (ESA) of the finished object? If we are allowed to drill two or three mutually orthogonal cylindrical holes of the same radius, which common radius will maximize the total ESA?
\end{abstract}

\section{Introduction}

We are three worms who live in Michelle's Craft Store that sells polystyrene foam balls and cubes. Naturally, we love to drill holes through these objects as we feed ourselves, while that very action upsets the store owner beyond crazy. Do you not believe that we can eat such things? (See [1]). The store owner prayed to save her business from us while we prayed to save our lives. Both of our prayers were answered simultaneously and affirmatively!

Metatron, the Angel of Mathematics, endowed us with a special skill to drill perfectly cylindrical holes of any radius we choose. Metatron blessed us: "You shall live as long as you abide by the following four requirements:

1) None of you drills more than one hole through any one object;

2) Each of you chooses an axis passing through the center of the object, and, if it is a cube, also passing through either a face-center $(\mathrm{F})$, or an edge-center $(\mathrm{E})$, or a vertex $(\mathrm{V})$;

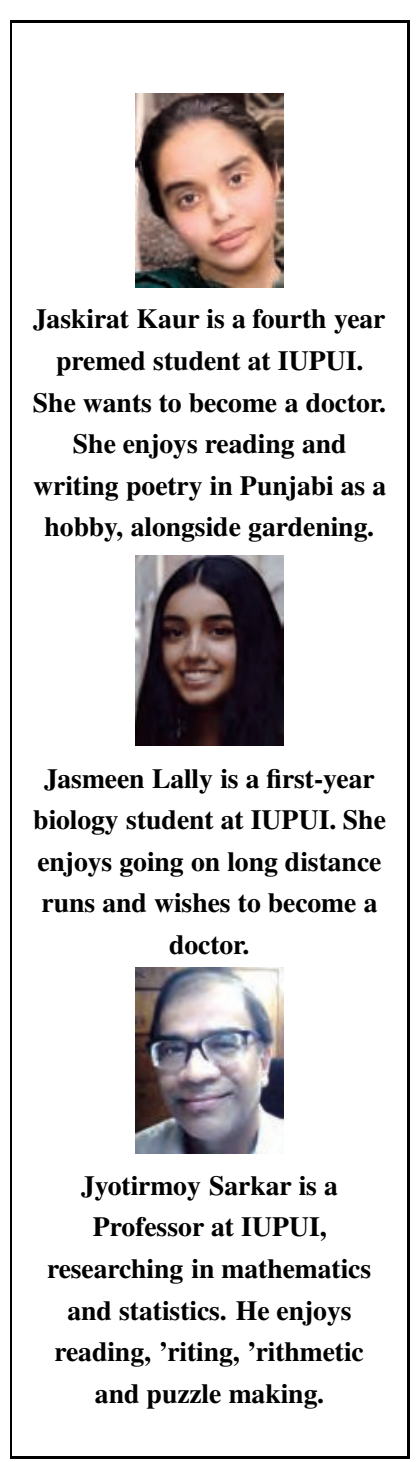

*Vol.26, No.4, DOI: https://doi.org/10.1007/s12045-021-1151-y 
Figure 1. Through a ball we drilled three holes. Through a cube we drilled cylindrical holes of type EF, $\mathrm{EE}$ and VE. Other types are also permissible. We are yet to determine the radius that will maximize the ESA. Can you do it?

\section{Keywords}

Radius of a ball, axis of a cylinder radius of a cylinder, plane cut, mutually orthogonal axes.

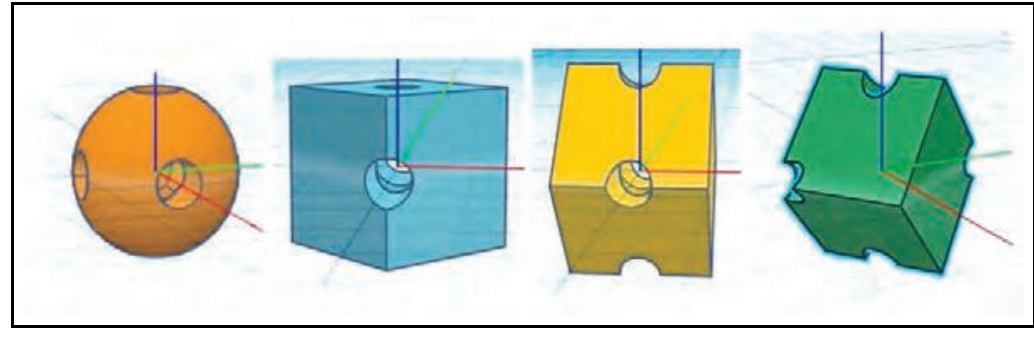

3) If two or three among you drill through the same object, then (3a) your multiple axes remain mutually orthogonal, and (3b) you choose the same radius; and

(4) Whether you drill alone or together, you always maximize the total exposed surface area (ESA) of the finished object."

Next, Metatron counseled the store owner:

"These drilled-through objects are fantastic educational specimens for a calculus class. Sell them at a premium price proportional to the total ESA."

The store owner and we are living happily ever after. Won't you please visit us and buy our marvelous products? Some sample works-in-progress are shown in Figure 1.

Can you determine the direction of the axis and the radius of the hole that we must choose when drilling alone? How about when we are drilling in pair, or in triplet? We had to answer these optimization questions lest we lose our lives! We strongly urge you to discover the answers on your own or in collaboration with a few like-minded curious partners. After you have tried long and hard, you may return to our answers given in Sections 2-4. Section 5 posses some follow-up open questions.

We used the freeware $\mathrm{R}$ for all calculations and Tinkercad for drawing all three-dimensional (3D) figures. You may proceed to read Section 1 now or return to it later. 


\section{Symmetry, Diameters, and Their Orthogonality}

Mathematical thinking is a great boon. It has helped us discover many features of the two 3D objects of our passion-the ball and the cube. We document some of these features relevant to answering our questions. To begin, let us choose a measurement scale and impose a Cartesian system of coordinates on each object.

\section{The Ball and Its Surface Area}

The unit ball has a center at $(0,0,0)$; and it is given by the set of points occupying 3D space no farther than one unit away (in the sense of the 3D Euclidean distance) from the center. That is, the unit ball is given by

$$
B_{3}=\left\{(x, y, z): x^{2}+y^{2}+z^{2} \leq 1\right\} .
$$

The boundary of the unit ball $B_{3}$ is the set of points exactly one unit distance away from the center; it is given by $S_{2}=\{(x, y, z)$ : $\left.x^{2}+y^{2}+z^{2}=1\right\}$. The boundary $S_{2}$ is called the unit sphere, which is a two-dimensional (2D) surface that lives in a $3 \mathrm{D}$ space.

The ESA (exposed surface area) of the unit ball (or the outer area of the unit sphere) equals the lateral (curved) surface area of the smallest right circular cylinder that contains the ball, which can be cut open into a rectangle of height 2 units and length $2 \pi$ units, and hence has area $4 \pi=12.56637$ unit $^{2}$. Conversely, the rectangle can be rolled and glued to form the lateral surface of the cylinder. See [2] for an elementary Euclidean geometric proof attributed to Archimedes. (For other applications of the method of Archimedes, see [3]). From the proof it is also evident that the curved surface area of a cap of the unit ball of height $h$, obtained by a plane cut at a distance $d=1-h$ from the center, is $2 \pi h$ unit $^{2}$.

\section{Diameters}

Any line segment passing through the centre and restricted within the ball (or terminated by the sphere) is called the diameter of the
Mathematical thinking has helped us discover many features of the ball and the cube.

ESA of a unit ball $=$ Lateral surface area of circumscribing cylinder $=$ Area of rectangle

Long before modern calculus was invented in the late 17 th century, Archimedes discovered the area of a sphere using geometry. 
Figure 2. The surface of a sphere equals the lateral surface of a circumscribing cylinder. Likewise, the surface of a cap of a sphere.

A globe models a ball: The north pole $\mathrm{N}$ and south pole $\mathrm{S}$ are obvious. If $B$ is the intersection of the international date line with the equator, where are $\mathrm{E}, \mathrm{F}$ and $\mathrm{W}$ ?

Can you determine the radius of each hole?

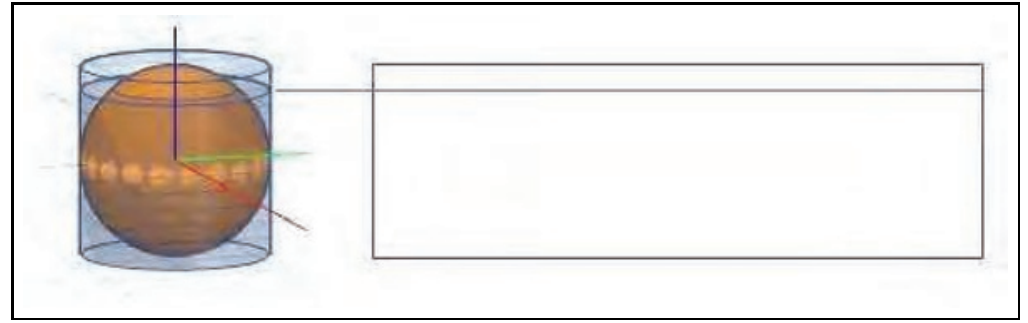

ball. All diameters of $B_{3}$ have the same constant length 2. Without loss of generality, the two endpoints of any diameter can be labelled as the north pole $N$ and the south pole $S$, respectively. Then the plane orthogonal to the $N S$ diameter is called the equatorial plane, and the intersection with the sphere is called the equator, a term borrowed from a globe, which serves to model a ball. Any diameter that lives in the equatorial plane is orthogonal to the $N S$ diameter and maybe labelled $E W$ (to denote east and west directions). A third diameter orthogonal to both $N S$ and $E W$ is labelled $F B$ (for front and back directions).

Metatron has permitted us to drill either one, or two, or three cylindrical holes with axes along diameters $N S, E W, F B$, without loss of generality. But it is up to us to determine the radius carefully so that we maximize the total ESA. Can you do so independently of us? Figure 3 shows our works-in-progress.

\section{The Cube and Its Surface Area}

The unit cube has a center at $(0,0,0)$; and it consists of the following set of points in 3-d space

$$
C_{3}=\{(x, y, z):-1 \leq x, y, z \leq 1\} .
$$

Thus, points in the cube have all three coordinates no farther than one unit away (in the sense of 1D distance) from 0 .

Note that our unit cube has all sides of length 2 (and not 1 as defined under different circumstances). The cube has six square faces each of side length 2. Hence, the total ESA of the cube is 24 unit $^{2}$. 


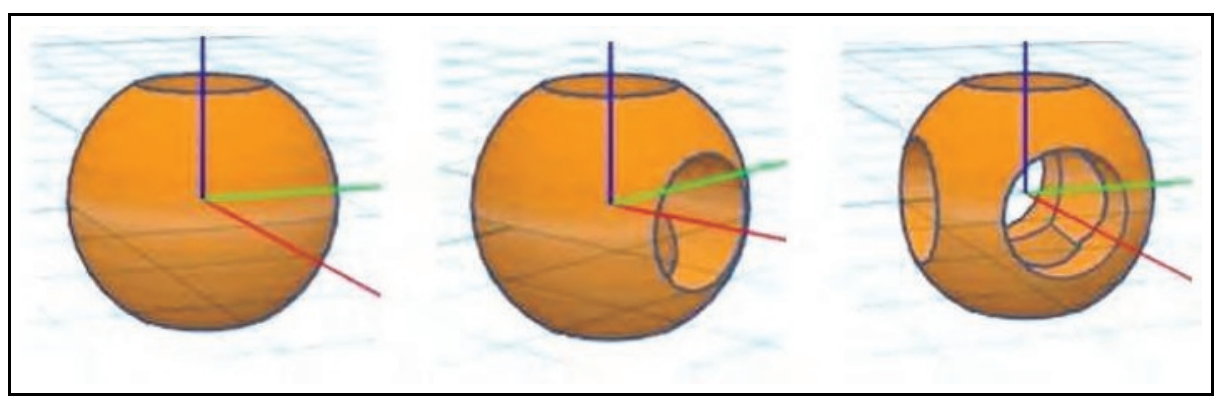

\section{Points of Tangency}

Given the above definitions, it is easy to see that $B_{3}$ is the largest ball that fits inside $C_{3}$, and $C_{3}$ is the smallest cube that covers $B_{3}$. Moreover, the boundaries of $B_{3}$ and $C_{3}$ are tangential to each other at the centers of the six faces of $C_{3}$.

\section{Symmetries}

We marvel at the symmetries exhibited by $B_{3}$ and $C_{3}$. By a symmetry, we mean a distance-preserving transformation such as a rotation or a reflection that maps an object onto itself. The unit cube $C_{3}$ has a $90^{\circ}$ rotation symmetry about any of the three axes and a reflection symmetry about any of the three Cartesian planes- $x y, y z, z x$, and also about any of the six planes $x=y, x=$ $-y, y=z, y=-z, z=x, z=-x$ that pass through four vertices of the cube. On the other hand, for a ball, any arbitrary rotation about the $x$-axis, and/or about the $y$-axis, and/or about the $z$-axis maps the ball onto itself. Also, a reflection about any plane passing through the centre maps the ball onto itself. Moreover, neither transformation changes the distance between two points. Therefore, the optimal radius of cylindrical holes through a ball depends on the number of holes, but not on their directions. Learn more about symmetry in mathematics from [5].
Figure 3. Our works-inprogress as we drill one, two or three mutually orthogonal cylindrical holes of the same radius. Which radius will maximize the ESA?

$B_{3}$ and $C_{3}$ are tangential to each other at six points $N, S, E, W, F, B$

A cube has only finitely-many rotation and reflection symmetries, a ball infinitely-many. 


\section{Diameters of a Cube}

The diameters of a cube are of varying lengths.

The shortest diameters pass through the face-centers of the cube, and are mutually orthogonal.

The longest diameters, also called the diagonals, pass through the vertices of the cube. No two diagonals are orthogonal to each other.

The plane orthogonal to a diagonal cuts the cube

to produce a regular hexagonal cross-section of side length $\sqrt{2}$.
For the ball $B_{3}$, all the diameters have the same constant length 2. Not so for the cube! Yes, the diameters of the cube are also line segments passing through the centre and restricted within the cube. However, they are of varying lengths. We mention below some special diameters of the cube.

\section{Shortest Diameters}

Each of the three shortest diameters, joining $(-1,0,0)$ to $(1,0,0)$, or $(0,-1,0)$ to $(0,1,0)$, or $(0,0,-1)$ to $(0,0,1)$, ends at some points of tangency between $B_{3}$ and $C_{3}$, which are the centers of the six faces of the cube. These three diameters are mutually orthogonal and have length 2 . We may choose one, two or all three of these diameters to form holes of types F, FF and FFF, respectively.

\section{Longest Diameters}

Each of the four longest diameters, joining $(-1,-1,-1)$ to $(1,1,1)$, or $(-1,-1,1)$ to $(1,1,-1)$, or $(-1,1,-1)$ to $(1,-1,1)$, or $(1,-1,-1)$ to $(-1,1,1)$, ends at opposite vertices of the cube.

They are also called diagonals of the unit cube. While they all have length $2 \sqrt{3}$, no two of these four diagonals are orthogonal to each other. In fact, any two of these four diagonals intersect each other at the centre making an acute angle measuring $2 \tan ^{-1} \sqrt{1 / 2}=0.3918266 \pi=1.230959=70.52878^{\circ}$. It should be noted that choosing two such diagonals as axes of the cylindrical holes would violate requirement (3a). Should we choose one such diagonal as an axis, the other one or two axes must lie in the plane through the centre orthogonal to the chosen diagonal, which plane produces a regular hexagonal cross-section of side length $\sqrt{2}$. In view of requirement (2), the second axis necessarily passes through an edge-centre. But then, a third axis is forced to pass through a point $M$, which is interior and non-central to a face. 
In fact, the coordinates of such an $M$ are the 24 permutations of $( \pm 1 / 2, \pm 1 / 2, \pm 1)$, four on each face. Therefore, while an axis of type $\mathrm{V}$ and two axes of type VE are permissible choices, a triplet of axes containing a type $\mathrm{V}$ is not permitted!

\section{Intermediate Diameters}

For any intermediate $d(2<d<2 \sqrt{3})$, there are infinitely-many diameters of length $d$, with endpoints on the intersection of $C_{3}$ and a sphere of diameter $d$. Among these, $d=2 \sqrt{2}$ deserves special attention since the middle points of the twelve edges of $C_{3}$ are at a distance $\sqrt{2}$ from the centre. By joining centrally opposite pairs of these middle points, we form six E-diameters: Pairs of these E-diameters live in the same Cartesian plane $x y, y z$, or $z x$; and the members within each pair are orthogonal (and form a hole of type EE); but they make a $\pi / 3=60^{\circ}$ angle with any of the four remaining E-diameters. Mixing these different sets of E-diameters that do not live in the same Cartesian plane violates requirement 3(a).

\section{Strategy to Solve Our Problem.}

For the unit ball $B_{3}$, it does not matter which points on the surface the axes emerge from since all diameters are interchangeable. But for the unit cube $C_{3}$, to satisfy requirement (2) of Metatron, the first axis must emerge from either the face-centre $F$ or the edgecentre $E$ or the vertex $V$ of the cube, yielding the three types of single cylindrical holes.

There are six possible types of two holes: EE, EF, EV, FF, FV, $\mathrm{VV}$; but the last two pairs are not permissible, since they violate requirement 3(a).

(We already reasoned that if the first axis is of type $\mathrm{V}$, then the second axis must be of type E.) Likewise, for three holes, not all the 10 combinations of types are permissible. For each of the four permissible pairs, the third axis is unique and yields only two triplets-FFF and EEF. Thus, 3 single-hole-, 4 double-hole-, and
Axes of types VF and VV are not permitted, nor are triplets containing a type $\mathrm{V}$.
E-diameters in the same Cartesian plane are orthogonal to each other. We may pick them to form a hole of type EE. 


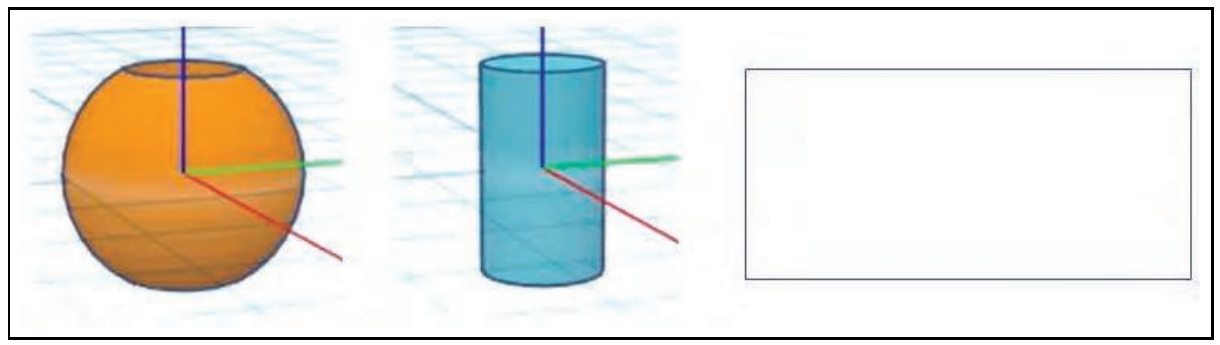

Figure 4. Drill one cylindrical hole through a ball. What is the optimal radius?
2 triple-hole configurations are permitted.

\section{SPOILER ALERT}

We are about to solve the optimization problems. Proceed only after you have given yourself enough opportunity to solve them.

For each of the nine permissible configurations, we must determine the optimal (common) radius $r$ of the cylindrical hole(s) that will maximize the total ESA of the drilled-through unit cube. We shall express the total ESA as a function of the radius and use single-variable calculus techniques to find the maximum ESA.

\section{Drilling Through the Ball}

The first cylindrical hole can be drilled in any direction through the origin. Without loss of generality, that chosen direction can be labelled as NS (blue axis). The second hole must have axis in the equatorial plane. Without loss of generality, we choose the $E W$ diameter as the second axis (green). Then the third hole necessarily has an axis (red) along the $F B$ diameter.

\section{One Hole}

If we unwrap the lateral surface of the cylindrical hole (imagine that we have made a paper model of a hollow cylinder) we get a rectangle of length $2 \pi r$ and height $2 \sqrt{1-r^{2}}$. Therefore, the total ESA of the unit ball with one cylindrical hole of radius $r$ equals the surface area of the unit ball, minus the area of the two caps 


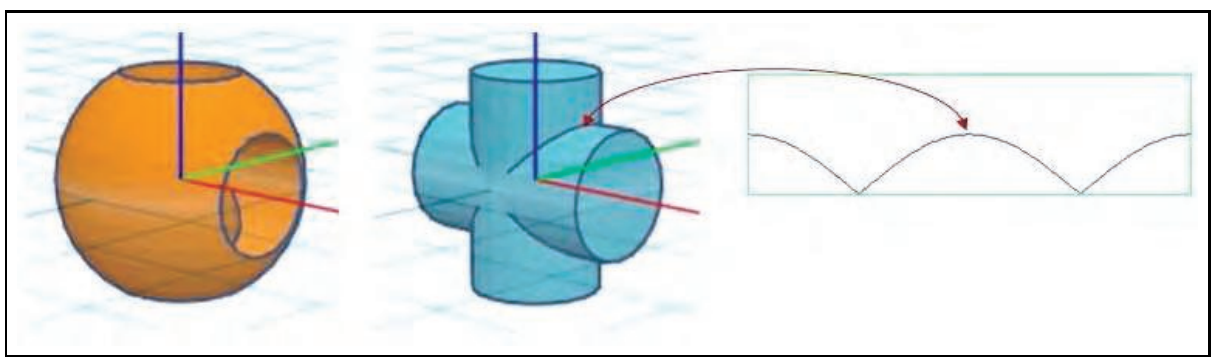

(one on top and one at the bottom) of height $\left[1-\sqrt{1-r^{2}}\right]$, plus the lateral area of the interior cylinder; that is,

$$
\begin{aligned}
A_{1}^{B}(r) & =4 \pi-2 \cdot 2 \pi\left[1-\sqrt{1-r^{2}}\right]+2 \pi r \cdot 2 \sqrt{1-r^{2}} \\
& =4 \pi(1+r) \sqrt{1-r^{2}} .
\end{aligned}
$$

The function (3) is continuous on $[0,1]$ and differentiable on $(0,1)$. Differentiating (3) with respect to $r$, using the product rule of differentiation, we have

$$
\frac{d}{d r} A_{1}^{B}(r)=4 \pi\left[\sqrt{1-r^{2}}+\frac{(1+r)(-\mathcal{R} r)}{\mathfrak{2} \sqrt{1-r^{2}}}\right]=4 \pi \frac{(1+r)(1-2 r)}{\sqrt{1-r^{2}}} .
$$

Thereafter, solving the first order condition, we get $r=1 / 2$. The other solution $r=-1$ is inadmissible, since a negative radius has no meaning. Furthermore, the second derivative evaluated at the only solution $r=1 / 2$ being $-2 \sqrt{3} \pi<0$, the objective function has been maximized. This principle can be found in any standard calculus textbook such as [4]. Substituting $r=1 / 2$ in (3), the maximum ESA is $3 \sqrt{3} \pi=5.196152 \pi=16.32419$ unit $^{2}$. Compared to a ball with no hole, the ESA is about $29.9 \%$ higher.

Henceforth, we will simply solve the first-order condition and leave it to the reader to verify that the reported solution indeed maximizes (and not minimizes) the objective function.

\section{Two Holes}

Consider two holes with axes $N S$ (blue) and $F B$ (red). Reverse the hole and the matter: Fill the holes with a solid and melt away
Figure 5. Drill two orthogonal cylindrical holes through a ball. What is the optimal common radius?

$\mathrm{ESA}=$ Ball -2 caps + cylinder

A principle of maximization: For a continuous function, a relative maximum occurs where the first derivative is zero and the second derivative is negative.

Print the parametric curve; cut along the graph; wrap and glue to make the model of a cylinder with two cuts. 
the ball (yielding the blue intersecting cylinders). Then take two plane cuts along the bisectors of the two axes orthogonal to the $N S-F B$ plane. Then the newly minted solid is cut up into four identical components.

Let us study any one of them, say the one closest to $N$. If we unwrap the lateral surface of the component (imagine a paper model of a hollow component), we get a rectangle of length $2 \pi r$ and height $\sqrt{1-r^{2}}$, minus a region bounded by the parametric curve $(r \theta, r|\cos \theta|)$ for $\theta \in(-\pi, \pi)$. Hence, this region has area

$$
4 \int_{0}^{\pi / 2} r \cos \theta \cdot r d \theta=4 r^{2}
$$

When two cylinders of radius $r$ intersect orthogonally, the area of the solid of intersection, using Cavalieri's principle, is $16 r^{2}$.

Furthermore, if these cylinders have a height $2 r$, then the total area of the curved surfaces of the union is also $16 r^{2}$.

$\mathrm{ESA}=$ ball -4 caps +4 cylindrical components (with cuts).

How to solve a cubic equation? See [7] a number free of $\pi$. How stunning! More surprisingly, you can prove this result without using calculus; simply use Cavalieri's principle (see [6]).

Therefore, the total ESA of the unit ball with two orthogonal cylindrical holes of radius $r$ equals the surface area of the unit ball, minus the area of the four caps (near $N, S, F, B$ points), plus four times the lateral area of the cylindrical component (with two plane cuts) closest to $N$; that is,

$$
\begin{aligned}
A_{2}^{B}(r) & =4 \pi-4 \cdot 2 \pi\left[1-\sqrt{1-r^{2}}\right]+4 \cdot\left[2 \pi r \cdot \sqrt{1-r^{2}}-4 r^{2}\right], \\
& =-4 \pi+8 \pi(1+r) \sqrt{1-r^{2}}-16 r^{2} .
\end{aligned}
$$

Differentiating (4) with respect to $r$, as was done to (3), we have

$$
\begin{aligned}
\frac{d}{d r} A_{2}^{B}(r) & =8 \pi\left[\sqrt{1-r^{2}}+\frac{(1+r)(-r)}{\sqrt{1-r^{2}}}\right]-32 r, \\
& =8 \pi \frac{(1+r)(1-2 r)}{\sqrt{1-r^{2}}}-32 r .
\end{aligned}
$$

The first order condition can be written as a cubic equation in $r$

$$
\pi^{2}(1+r)(1-2 r)^{2}=16(1-r) r^{2},
$$

which has three real solutions: $-0.746,0.346,0.688$. Evaluating (4) at the positive solutions and zooming in, we get $r=0.34637$ 


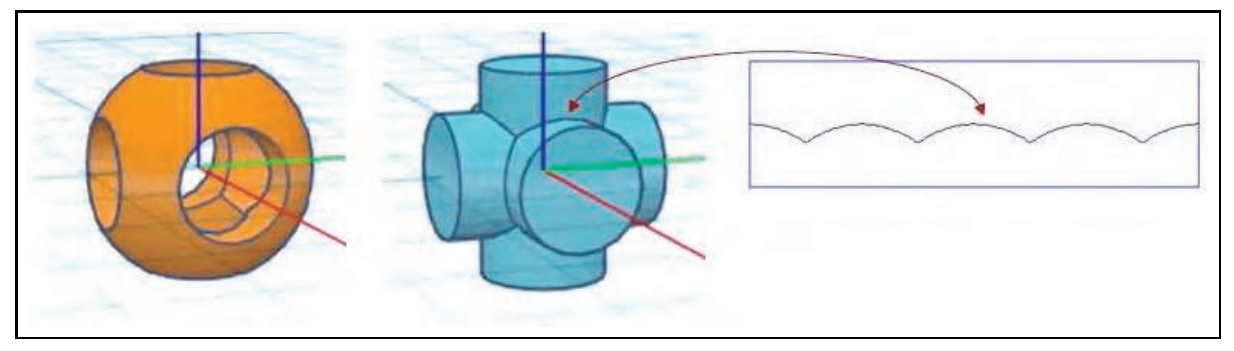

at which the maximum ESA is 17.2574 unit $^{2}$. Interested readers may learn how to solve a cubic equation from [7].

Thus, compared to one cylindrical hole, when we drill two orthogonal cylindrical holes, we do not double the newly-generated interior surface area: First, we lose some surface at the overlap of the cylinders; and second, we actually reduce the common radius by about $30.6 \%$ (from 0.5 to 0.34647 ). Nonetheless, we increase the total ESA by $5.7 \%$. Furthermore, compared to a ball with no hole, the surface area increases by $37.3 \%$.

\section{Three Holes}

Once again, fill the holes with solid material and melt away the polystyrene ball. The three holes with axes $N S, E W$ and $F B$ are composed of six identical components. One such component closest to $N$ may be constructed by cutting further the $N$ component of the previous two-hole case with two plane cuts that are $90^{\circ}$ rotations about the $N S$ axis of the previous two cuts.

If we unwrap the lateral surface of this modified $N$-component (imagine once again that we have made a paper model of a hollow component), we get a rectangle of length $2 \pi r$ and height $\sqrt{1-r^{2}}$, minus a region bounded by the curve $(r \theta, r \max \{|\cos \theta|,|\sin \theta|\})$ for $\theta \in(-\pi, \pi)$. Hence, this region has an area

$$
8 \int_{0}^{\pi / 4} r \cos \theta \cdot r d \theta=4 \sqrt{2} r^{2}
$$

By now it comes as no surprise that this area is also free of $\pi$.
Figure 6. Drill three mutually orthogonal cylindrical holes through a ball. What is the optimal common radius?

Two holes do not double the internal surface!
Print the parametric curve; cut along the graph; wrap and glue to make the model of a cylinder with four cuts. 
$\mathrm{ESA}=$ Ball -6 Caps +6 Cylindrical components (with 4 cuts).

Compare the maximal total ESA of a unit ball with 3 holes with that of a ball with fewer holes.

There are three types of single holes: E, F, V.
Therefore, the total ESA of the unit ball with three mutually orthogonal cylindrical holes of radius $r$ equals the surface area of the unit ball, minus the area of the six caps (near $N, S, E, W, F, B$ points) of height $\left[1-\sqrt{1-r^{2}}\right]$, plus six times the lateral area of the above described $N$-component; that is,

$$
\begin{aligned}
A_{3}^{B}(r) & =4 \pi-6 \cdot 2 \pi\left[1-\sqrt{1-r^{2}}\right]+6 \cdot\left[2 \pi r \cdot \sqrt{1-r^{2}}-4 \sqrt{2} r^{2}\right] \\
& =-8 \pi+12 \pi(1+r) \sqrt{1-r^{2}}-24 \sqrt{2} r^{2}
\end{aligned}
$$

Differentiating (5) with respect to $r$, as twice before, we have

$$
\begin{aligned}
\frac{d}{d r} A_{3}^{B}(r) & =12 \pi\left[\sqrt{1-r^{2}}+\frac{(1+r)(-r)}{\sqrt{1-r^{2}}}\right]-48 \sqrt{2} r \\
& =12 \pi \frac{(1+r)(1-2 r)}{\sqrt{1-r^{2}}}-48 \sqrt{2} r
\end{aligned}
$$

Again, solving the first order condition numerically, we get $r=$ 0.30135 , substituting which in (5), the maximum ESA is 18.56413 unit $^{2}$.

Thus, when we drill three mutually orthogonal cylindrical holes, compared to two orthogonal cylindrical holes, the common radius reduces further by $13.0 \%$ (from 0.34647 to 0.30135 ); but the total ESA increases by $7.6 \%$. Compared to one cylindrical hole, the radius decreases by $39.5 \%$, but the total ESA increases by $13.7 \%$. Finally, compared to a unit ball with no hole, the total ESA has increased by $47.7 \%$.

\section{Drilling One Hole Through a Cube}

As discussed in Section 1, there are three types of a single cylindrical hole that satisfy requirement (2) of Metatron: E, F and V. We shall deduce the optimal radius for each type.

\section{One Hole of Type F}

The total ESA of the unit cube with one cylindrical hole of type $\mathrm{F}$ and radius $r \leq 1$ equals the surface area of the unit cube, minus 


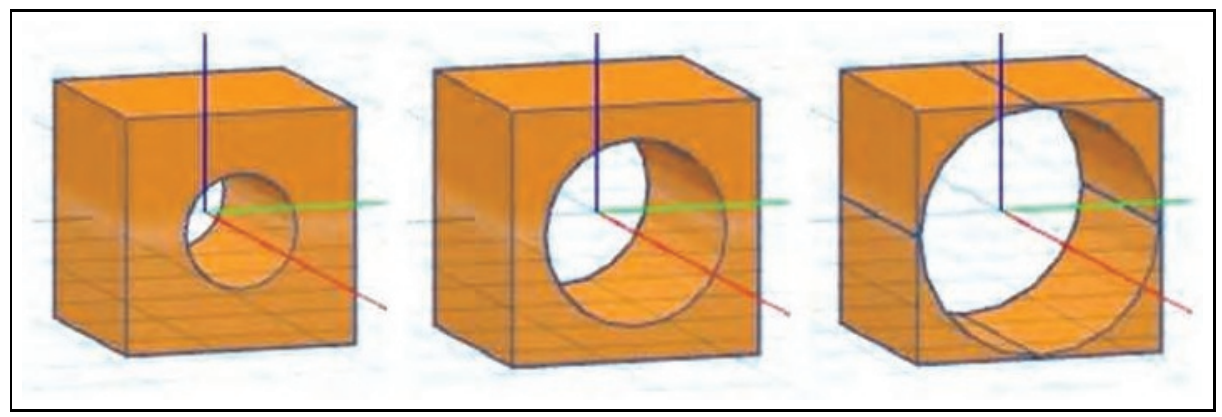

the area of the two circular discs (one on top and one at the bottom face of the cube) of radius $r$, plus the lateral area of the cylinder; that is,

$$
A_{F}^{C}(r)=24-2 \cdot \pi r^{2}+2 \cdot 2 \pi r
$$

Differentiating (6) with respect to $r$, we have

$$
\frac{d}{d r} A_{F}^{C}(r)=-4 \pi r+4 \pi
$$

Figure 7. Drill one cylindrical hole of type $\mathrm{F}$ through a unit cube. What is the optimal radius?

ESA $=$ Cube -2 Discs

+ Cylindrical hole.

Thereafter, solving the first-order condition, which is a linear equation, we get $r=1$. We should note that a hole of type $\mathrm{F}$ and radius $r=1$ brings the cube on the verge of falling apart. See the rightmost picture in Figure 7. The store owner will act wisely to wrap the polystyrene foam cube inside a box that we won't be tempted to drill through.

Note also that (6) is defined only for $r \in[0,1]$, and its derivative is strictly positive on $(0,1)$ with its left limit 0 at $r=1$. In fact, if we imagine a cylindrical hole of radius $r>1$, the cube has already split into four components, and any increase in the radius decreases the area of each of the five faces of the components; hence, it also decreases the total ESA. Moreover, $A_{F}^{C}(1+)=A_{F}^{C}(1)$. Therefore, indeed the total ESA is maximized at $r=1$, substituting which in (6), the maximum ESA is $24+2 \pi=$ 30.28319 unit $^{2}$. Compared to a cube with no hole, the total ESA has increased by about $26.2 \%$.

$r=1$ brings the cube on the verge of falling apart.

If $r>1$, the cube has already split into four components! 


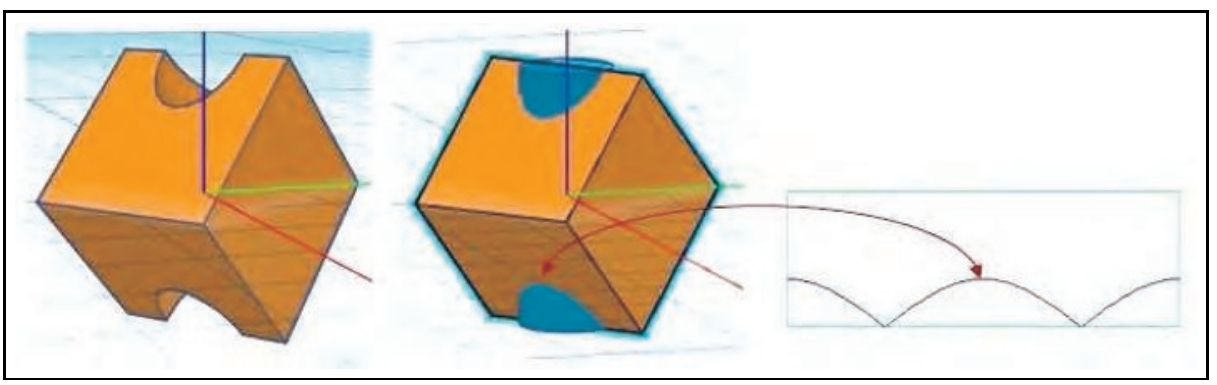

Figure 8. Drill one cylindrical hole of type E through a unit cube. What is the optimal radius?

Total ESA = Unit cube - 4 Half-ellipses +2 Sliced-off cylinders.
One Hole of Type E

Suppose that we drill a hole of type E through a cube. Now imagine that we replace the hole with a new solid and then remove the remaining part of the cube. Further, impose a plane cut through the centre orthogonal to the axis of the new solid. This gives us two identical, almost cylindrical solids, but with one end sliced off by two plane faces of the cube. These plane faces make $\pi / 4=45^{\circ}$ angles with the axis of the new solid and meet each other at the same diameter as the base. Each plane slice exposes a region in the shape of an ellipse with minor-axis $r$ and major-axis $\sqrt{2} r$ cut in half along the minor axis.

If we make a paper model of the lateral surface of the lower half-cylinder and unwrap it, we get a rectangle of length $2 \pi r$ and height $\sqrt{2}$, with a bottom region sliced off by the parametric curve $(r \theta, r|\cos \theta|)$ for $\theta \in(-\pi, \pi)$. Recall from the previous section that the area below this curve is $4 r^{2}$.

Therefore, the total ESA of the unit cube with one cylindrical hole of type $\mathrm{E}$ and radius $r$ equals the surface area of the unit cube, minus the area of four half-ellipses (two on the two cube-faces adjacent to one edge and two on the two cube-faces adjacent to the opposite edge) of minor-axis $r$ and major-axis $\sqrt{2} r$, plus twice the lateral surface area of the above described sliced-off half-cylinder; that is,

$$
\begin{aligned}
A_{E}^{C}(r) & =24-2 \cdot \sqrt{2} \pi r^{2}+2\left[2 \sqrt{2} \pi r-4 r^{2}\right], \\
& =24-2 \sqrt{2} \pi r^{2}+4 \sqrt{2} \pi r-8 r^{2} .
\end{aligned}
$$




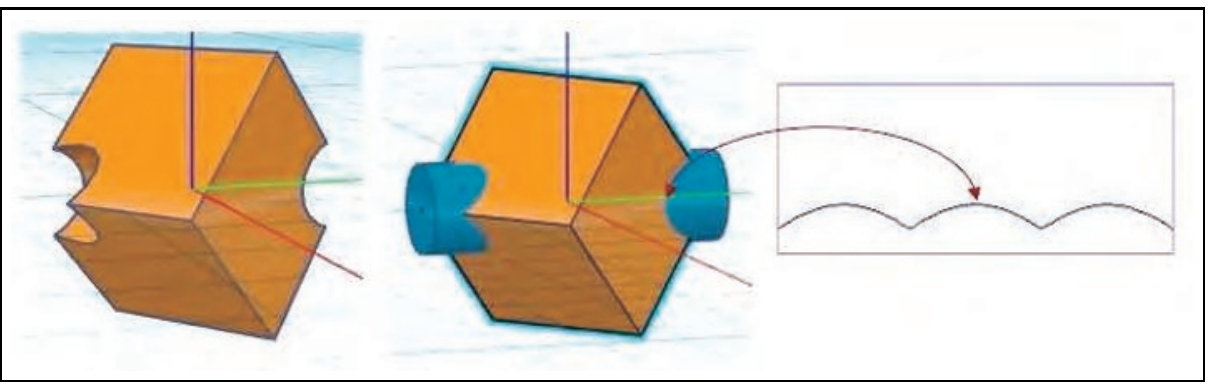

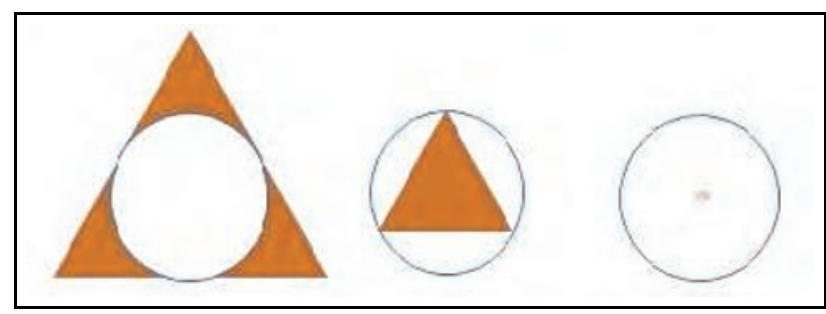

Differentiating (7) with respect to $r$, we have

$$
\frac{d}{d r} A_{E}^{C}(r)=-4 \sqrt{2} \pi r+4 \sqrt{2} \pi-16 r .
$$

Solving the (linear) first-order condition, we get the optimal radius as $r=\pi /(\pi+2 \sqrt{2})=0.52623$. Note that this solution is less than 1, so that the cube still remains intact. Substituting this solution in (7), the maximum ESA is 28.67594 unit $^{2}$. Compared to a cube with no hole, the total ESA of a cube with one type E hole has increased by about $19.5 \%$.

Therefore, for a single hole, between a type $\mathrm{F}$ and a type $\mathrm{E}$, the store owner prefers type F. Intuitively, although a type E 'cylindrical' hole (with axis length $2 \sqrt{2}$ ) has an axis longer than that of a type $\mathrm{F}$ hole (with axis length 2), the former has a much smaller radius than the latter. The optimal type $\mathrm{F}$ hole makes the total ESA about 5.6\% larger than that made by the optimal type E hole.
Figure 9. Drill one cylindrical hole of type $\mathrm{V}$ through a unit cube. What is the optimal radius?

Figure 10. Three crosssections of the half-cylinder of length $\sqrt{3}$ and radius $r$, before it is sliced off by the three faces of the cube.

Linear FOC again! Are we lucky, or what?

ESA $(\mathrm{F})>$ ESA (E). 


\section{One Hole of Type V}

Having drilled a hole of type $\mathrm{V}$ through a cube, let us reverse the roles of the hole and the remaining solid. Equivalently, consider a solid cylinder of length $2 \sqrt{3}$ and radius $r$, and take a plane cut through the centre orthogonal to the axis to get two identical cylindrical solids of height $\sqrt{3}$. Let us study the right half of the cylinder shown in Figure 9 before it is sliced off by the three plane faces of the cube meeting at a vertex.

The rightmost end (towards the vertex of the cube) of this new cylindrical solid has a cross-sectional circle passing through the vertex of the cube (see Figure 10-right panel). A second crosssectional circle, $r / \sqrt{2}$ away from the rightmost end, cuts off from the unit cube a pyramid with an apex at the same vertex, three lateral sides of length $\sqrt{3 / 2} r$ each, and an equilateral triangular base of side length $\sqrt{3} r$ (see Figure 10-middle panel) . A third crosssectional circle, $\sqrt{2} r$ away from the rightmost one, is internally tangent to the base of a larger pyramid (double the first pyramid in every dimension) with apex at the same vertex, with three lateral sides of length $\sqrt{6} r$ each and an equilateral triangular base of side length $2 \sqrt{3} r$ (see Figure 10-left panel). The midpoint of any side of the base of the second pyramid is $\sqrt{3} r$ away from the vertex.

Next, the right half-cylindrical solid is sliced off by the three faces of the cube. Let us consider these cuts sequentially. The first face cuts the cylinder slantwise to from an elliptical cross-section of minor-axis $r$ and major-axis $\sqrt{3} r$. However, after the second and the third faces also cut the cylinder, only one-third of this elliptical cross-section remains on the right half-cylinder. It has area $(1 / 3) \sqrt{3} \pi r^{2}=\pi r^{2} / \sqrt{3}$, which is also the area lost by the other two faces of the cube around the vertex.

If we unwrap the lateral surface of this right half cylindrical solid after it has been cut by the three faces of the cube (see Figure 9

Make a paper model... after carefully sketching the periodic graph. again), we get a rectangle of length $2 \pi r$ and height $\sqrt{3}$, with a bottom region sliced off by the parametric curve $(r \theta, \sqrt{3} r|\cos \theta|)$ for $\theta \in(-\pi / 3, \pi / 3)$. Thereafter, the curve is periodically repeated 
on intervals $(-\pi,-\pi / 3)$ and $(\pi / 3, \pi)$. The area of this region over the interval $(-\pi, \pi)$ is

$$
6 \int_{0}^{\pi / 3} \sqrt{3} r \cos \theta r d \theta=9 r^{2}
$$

Therefore, the total ESA of a unit cube with a type V hole and radius $r$ equals the surface area of the unit cube, minus the area Free of $\pi$ ! By now we are accustomed to it. of six one-third-ellipses of minor-axis $r$ and major-axis $\sqrt{3} r$, plus twice the lateral area of the above described half-cylinder; that is,

$$
\begin{aligned}
A_{V}^{C}(r) & =24-6 \cdot \pi r^{2} / \sqrt{3}+2\left[\sqrt{3} \cdot 2 \pi r-9 r^{2}\right], \\
& =24-2 \sqrt{3} \pi r^{2}+4 \sqrt{3} \pi r-18 r^{2} .
\end{aligned}
$$

Differentiating (8) with respect to $r$, we have

$$
\frac{d}{d r} A_{V}^{C}(r)=-4 \sqrt{3} \pi r+4 \sqrt{3} \pi-36 r .
$$

Solving the first order condition, which is once again linear, we get $r=\pi /(\pi+3 \sqrt{3})=0.37679)$. Check that this optimal solution is less than $\sqrt{2}=1.4142$, the circum-radius of a regular hexagon of side length $\sqrt{2}$, so that the cube still remains intact. Substituting this solution in (8), the maximum ESA is 28.10055 unit $^{2}$. Compared to a cube with no hole, the total ESA of a cube with one type $\mathrm{V}$ hole has increased by about $17.1 \%$.

Therefore, in terms of ESA, we may write $\mathrm{F}>\mathrm{E}>\mathrm{V}$. In particular, among single holes of type F, E, and V, the store owner prefers type $\mathrm{F}$. Intuitively, although a type $\mathrm{V}$ cylindrical hole has an average axis-length bigger than the corresponding length 2 of a type $\mathrm{F}$ hole, the former has a much smaller radius than the latter. Compared to the optimal type $\mathrm{V}$ hole, the optimal type $\mathrm{E}$ hole has about 2\% larger ESA, and the optimal type F hole has $7.8 \%$ larger ESA.

\section{Drilling Two or Three Holes through a Cube}

We already reasoned in Section 1 that Metatron's requirements permit us to drill two orthogonal holes of types FF, FE, EE and

The derivations follow similar lines of reasoning. 


\begin{tabular}{|c|c|c|c|}
\hline \multirow{2}{*}{$\begin{array}{r}\text { Type of } \\
\text { holes }\end{array}$} & \multicolumn{2}{|c|}{ Total exposed surface area: } & \multirow{2}{*}{$\begin{array}{l}\text { Optimal } \\
\text { radius }\end{array}$} \\
\hline & linear term (in $r$ ) & quadratic term & \\
\hline $\mathrm{F}$ & $24+4 \pi r$ & $-2 \pi r^{2}$ & 1 \\
\hline $\mathrm{E}$ & $24+4 \sqrt{2} \pi r$ & $-2[\sqrt{2} \pi+4] r^{2}$ & $\pi /[\pi+2 \sqrt{2}]$ \\
\hline $\mathrm{V}$ & $24+4 \sqrt{3} \pi r$ & $-2[\sqrt{3} \pi+9] r^{2}$ & $\pi /[\pi+3 \sqrt{3}]$ \\
\hline FF & $24+8 \pi r$ & $-4[\pi+4] r^{2}$ & $\pi /[\pi+4]$ \\
\hline FE & $24+4(\sqrt{2}+1) \pi r$ & $-2[(\sqrt{2}+1) \pi+12] r^{2}$ & $\pi /[\pi+12(\sqrt{2}-1)]$ \\
\hline $\mathrm{EE}$ & $24+8 \sqrt{2} \pi r$ & $-4[\sqrt{2} \pi+8] r^{2}$ & $\pi /[\pi+4 \sqrt{2}]$ \\
\hline VE & $24+4(\sqrt{3}+\sqrt{2}) \pi r$ & $-2[(\sqrt{3}+\sqrt{2}) \pi+21] r^{2}$ & $\pi /[\pi+21(\sqrt{3}-\sqrt{2})]$ \\
\hline FFF & $24+12 \pi r$ & $-6[\pi+4 \sqrt{2}] r^{2}$ & $\pi /[\pi+4 \sqrt{2}]$ \\
\hline EEF & $24+4(1+2 \sqrt{2}) \pi r$ & $-2[(1+2 \sqrt{2}) \pi+12 \sqrt{2}] r^{2}$ & $\pi /[\pi+12(4-\sqrt{2}) / 7]$ \\
\hline
\end{tabular}

Table 1. Formulas for the total ESA and the optimal radius for various types of holes through a cube.

It surprised us that although $\mathrm{F}>\mathrm{E}>\mathrm{V}$, $\mathrm{EE}>\mathrm{VE}>\mathrm{EF}>\mathrm{FF}$.

FFF $>$ EE; but $r_{\mathrm{FFF}}=r_{\mathrm{EE}}$
VE, and three mutually orthogonal holes of types FFF and EEF. Since the derivations of the optimal radius follow similar lines of reasoning, utilizing quantities established in the previous two sections, we present the formulas for the total ESA and the optimal radius in Table 1, leaving details to the reader. For easy comparison, we also augment formulas (6)-(8) for one hole.

Numerical values of the optimal radius and maximum total ESA are shown in Table 2, and the diagrams of the finished products in Figure 11, followed by some commentaries.

Interestingly, while the optimal radius $r_{F}=1$ causes the unit cube to almost break, $r_{E}=.52623$ is much smaller than 1 , and $r_{V}=.37679$ is far short of $\sqrt{2}=1.4142$, values at which the unit cube is at risk of splitting.

It surprised us that a type E hole produces a $2 \%$ larger ESA than a type $\mathrm{V}$ hole, even though the former has an initial axis length $\sqrt{2}$, which is $19.3 \%$ smaller than that of the latter's $\sqrt{3}$. The reason, of course, is that the $r_{V}$ is $28.4 \%$ smaller than $r_{E}$. Likewise, we found it counter-intuitive that two holes of type EE produce a slightly larger ESA than two holes of type VE. Moreover, for a single hole, a type $\mathrm{F}$ attains a $5.6 \%$ higher ESA than a type E; but for two holes, a type FF falls $2.7 \%$ short of a type EE! Strangely, although type FFF has a 1.3\% higher ESA than type 
GENERAL ARTICLE

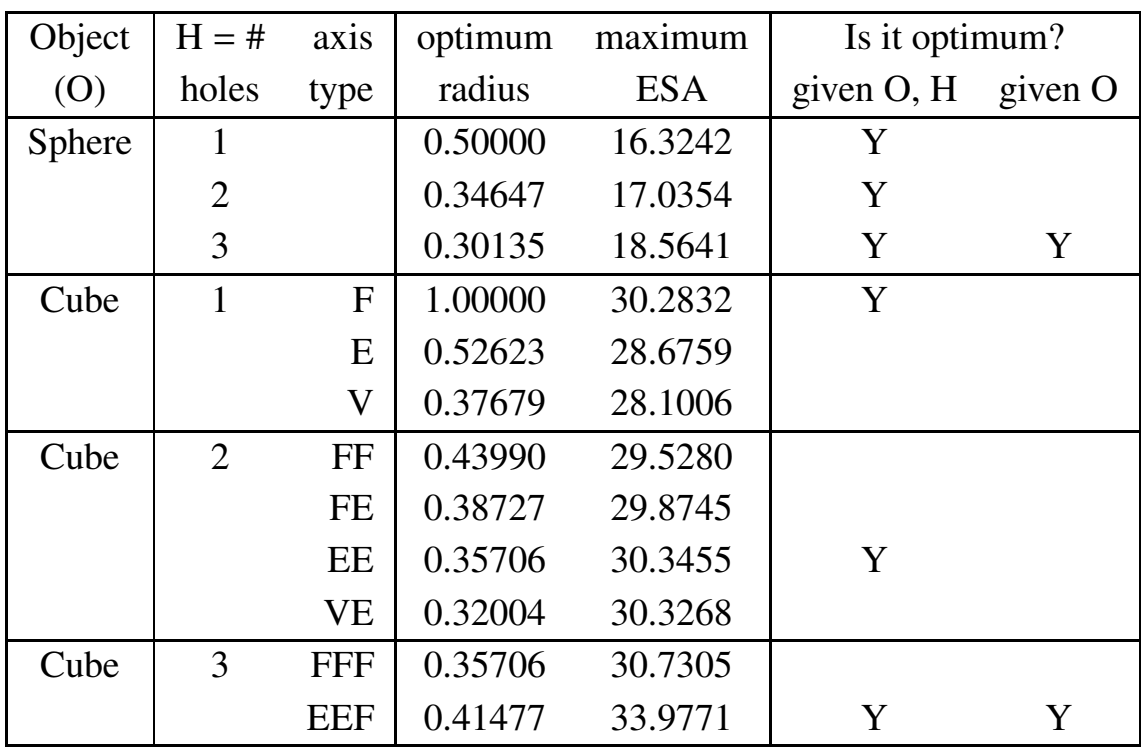

Table 2. Optimal radius for various types of holes.

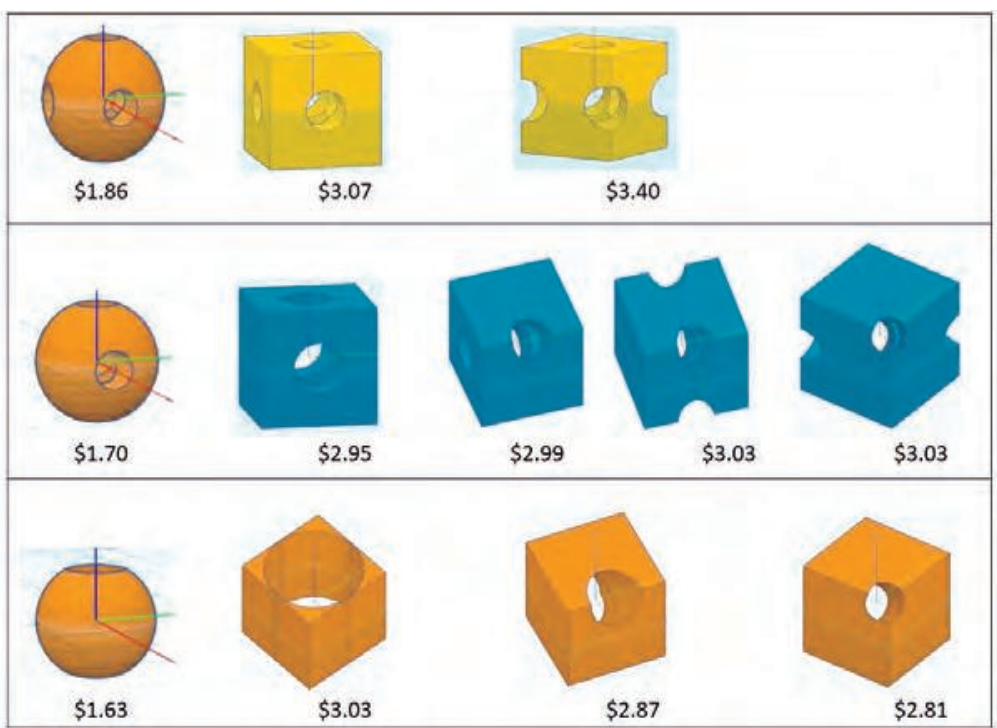

EE, the optimal radius for these two types are identical!

As for a unit ball, so for a unit cube, as the number of holes in-
Figure 11. Balls and cubes for sale at Michelle's Craft Store: Buy all 12 for $\$ 28.49$; that is, at a $12 \%$ discount. 
As anticipated, we do find EEF > FFF.

$\mathrm{EEF}>\mathrm{EE}$

We have a negotiation advantage over the store owner! Thank you, Archangel Metatron. creases, the corresponding optimal radius decreases, but the maximal total ESA increases. When we drill through a unit cube, if we choose only one hole, type $\mathrm{F}$ with optimal radius 1 is the best; if we choose two holes then type EE with $r_{\mathrm{EE}}=\pi /[\pi+4 \sqrt{2}]=$ 0.35706 maximizes the total ESA; and if we choose three holes then type EEF with $r_{\mathrm{EEF}}=\pi /[\pi+12(4-\sqrt{2}) / 7]=0.41447$ maximizes the total ESA, exceeding that of FFF by $10.6 \%$.

In summary, when drilling through a unit cube, when the number of holes increases from 1 to 2 , the corresponding optimal radius for the best type of holes decreases, but the total ESA increases by a minuscule $0.2 \%$. Also, unlike the single hole of type F, which almost fragments the unit cube, the two holes of type EE keep the cube intact. But when the number of holes increases from 2 to 3 , the optimal radius of type EEF is $17 \%$ larger than that of type EE and the total ESA increases by about $12.0 \%$.

There is yet another interesting feature of these optimal solutions that give us, lowly creatures, a negotiation advantage over the store owner: After we have drilled two optimal holes of type EE through a cube, in exchange for some nice treats from the store owner, we can modify the object by drilling a third hole of type $\mathrm{F}$ and then increasing the common radius of all three holes by about $16 \%$ and ESA by about $12 \%$. Likewise, after we have drilled two optimal holes of type EF through a cube, we can drill a third hole of type $\mathrm{E}$ and increase the common radius of all three holes by about $7.1 \%$ and ESA by about $13.7 \%$. This optimal hole of type EEF achieves the largest total ESA and hence is preferred by the store owner for she can sell it at the highest premium price. The total ESA of the cube with holes of type EEF is $41.7 \%$ higher than a unit cube with no holes.

\section{More Problems}

One solution opens the doors to more mathematics problems.
Oftentimes, when we find a solution to a mathematics problem, it opens the doors to more mathematics problems! Having solved the optimal radius problem subject to Metatron's four conditions, we have opened up a floodgate of other variations on the theme. 


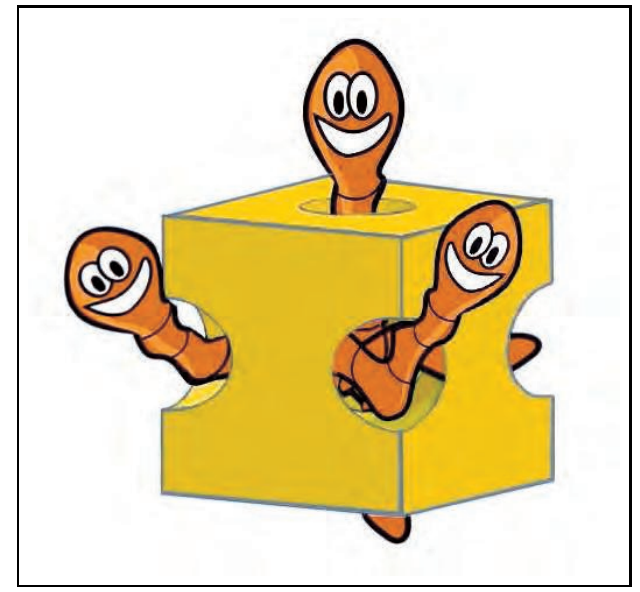

Here we list a few, beckoning the reader to wrestle with them:

1. Change the object through which to drill. Drill cylindrical holes through other Platonic solids (tetrahedron, octahedron, dodecahedron and icosahedron) of unit dimension, or Archimedes' cylinder that circumscribes a unit ball shown in Figure 2, or any other favourite convex object.

2. Change the objective function. Instead of maximizing the total ESA, you may want to maximize the total new surface area (NSA) generated by drilling the holes. That is, imagine that you have coloured red the outer surface of the object. As you keep increasing the radius of the cylindrical hole, the area of the red zone surely decreases and the newlyemerging colourless area increases for a while and thereafter decreases. When does it reach a maximum?

3. Change the type of hole. Metatron equipped us to drill cylindrical holes with a circular cross-section. Now drill holes with other cross-sectional shapes such as an ellipse (with a given ratio of the major axis to the minor axis) or a regular polygon (such holes are called prisms.) Thereafter, choose your favourite object and drill a number of holes of optimal dimension to maximize the total ESA or NSA.
Figure 12. Michelle gives us a special treat every time we make holes of type EEF.

Drill through your favourite objects.

The objective function is your subjective choice.

Drill holes with a different cross-section. 
What if three mutually orthogonal holes of type VEM are permitted? The alert reader can deduce the optimal radius using the results in this paper.
4. Change some of the requirements. Metatron imposed on us (or rather favoured us with) some requirements to abide by. We wish to get a waiver in one situation: Having chosen the first and the second holes of types $\mathrm{V}$ and $\mathrm{E}$ respectively, since the direction of the third hole is already determined by requirement 3(a), we could humbly request a deferral of requirement 2 for the third hole, allowing it to be of type $M$ discussed in Section 1.

If permitted, then drilling three mutually orthogonal holes of type VEM would result in an optimal radius of

$$
\pi\left[\pi+\frac{13+24 \sqrt{2}}{\sqrt{3}+\sqrt{2}+\sqrt{3 / 2}}\right]^{-1}=0.2263267
$$

and a total ESA of 31.54762 unit $^{2}$. Thus, a cube with three mutually orthogonal holes of type VEM has the narrowest holes among all configurations studied in this paper, yet it has the second-highest total ESA!

If you wish to change one or more of the requirements, please proceed with caution: For you may enter the realm of multivariate calculus.

\section{Acknowledgement}

We are thankful for the chance to convert a lemon (mandatory home quarantine due to COVID-19 pandemic) into a lemonade (learning mathematics and writing a paper).

\section{Suggested Reading}

[1] Mealworms can eat toxic polystyrene foam safely, Modern Farmer, modernfarmer.com/2020/01/mealworms-can-eat-toxic-styrofoam-safely/

[2] Archimedes and the area of sphere, Mathematics@CUHK, cuhkmath.wordpress.com/2018/01/05/archimedes-and-the-area-of-sphere/

[3] Tom M. Apostol and Mamikon A. Mnatsakanian, A fresh look at the method of Archimedes, The American Mathematical Monthly, Vol.111, No.6, pp.496-508, 2004.

[4] Marvin Bittinger, Neal Brand and John Quintanilla, Calculus for the Life Sciences, 2006, New York: Pearson Education, Inc. 
GENERAL ARTICLE

[5] Symmetry in Mathematics, Wikipedia, the free Encyclopedia, en.wikipedia.org/wiki/Symmetry_in_mathematics

[6] Cavalieri's principle, Wikipedia, the free Encyclopedia, https://en.wikipedia.org/wiki/Cavalieri\%27s_principle

[7] Eric W. Weisstein, Cubic formula, MathWorld-A Wolfram Web Resource, https://mathworld.wolfram.com/CubicFormula.html

Address for Correspondence Jaskirat Kaur $^{1}$ Jasmeen Lally ${ }^{2}$ Jyotirmoy Sarkar ${ }^{3}$ Indiana University-Purdue University Indianapolis

School of Science 402 N Blackford Street Indianapolis, IN 46202, USA Email: jk145@iu.edu ${ }^{1}$ jklally@iu.edu ${ }^{2}$ jsarkar@iupui.edu ${ }^{3}$ 